\title{
Airport Remote Tower Sensor Systems
}

\author{
Richard Papasin, Yuri Gawdiak, David A. Maluf, Christopher Leidich \\ NASA Ames Research Center \\ Peter B. Tran \\ QSS Group. Inc.
}

Copyright $\odot 2001$ Society of Automotive Engineers, Inc.

\begin{abstract}
Remote Tower Sensor Systems are proof-of-concept prototypes being developed by NASAAmes Research Center (NASAARC) with collaboration with the FAA and NOAA. RTSS began with the deployment of an Airport Approach Zone Camera System that includes real-time weather observations at San Francisco International Airport. The goal of this research is to develop, deploy, and demonstrate remotely operated cameras and sensors at several major airport hubs and un-towered airports. RTSS can provide real-time weather observations of airport approach zone. RTSS will integrate and test airport sensor packages that will allow remote access to realtime airport conditions and aircraft status.
\end{abstract}

\section{INTRODUCTION}

Airport remote tower sensor systems have been developed and fielded by NASAARC in collaboration with the Federal Aviation Administration (FAA) and the National Oceanic Atmospheric Administration (NOAA). These systems have initially been deployed at the San Francisco International Airport (SFO), the San Carlos Airport (SQL) and the Seattle-Tacoma International Airport (SEA). This initial design was called the Airport Approach Zone Camera System (AAZCS). A next generation design, called the Remote Tower Sensor System (RTSS) has been developed recently and will soon be deployed at the Half Moon Bay Airport (HAF) and a version of it will eventually be implemented at the Los Angeles International Airport (LAX). Figure 1 shows the overall configurations of the AAZCS and RTSS. Web based camera viewing and control is accomplished through a server located at NASAARC. For SFO, SQL and SEA image archiving is achieved at each location via Apple Macintosh servers. Eventually image archiving will occur at NASANARC for these sites. For HAF and LAX, archiving of image data as well as weather sensor data will all occur at NASANARC.

\section{AAZCS DEVELOPMENT}

Airport remote tower sensor systems began with the development and deployment of an Airport Approach Zone Camera System (AAZCS). The Aviation Surface Technologies Area Team, a part of the Computational Sciences Division of NASANAmes Research Center, developed the AAZCS in collaboration with the Center Weather Service Unit (CWSU) in early 1998 fueled by the concerns raised by the Meteorologist-In-Charge at the CWSU regarding the lack of real-time weather images from San Francisco International Airport (SFO).

Forecast of the onset and/or dissipation of low clouds are of major concern to the CWSU, FAA, and the aviation community. Low clouds prevent aircraft from landing simultaneously on the two primary runways at SFO because the runways are spaced much closer together than at other airports. The inability to implement dual approaches decreases the airport arrival rate by about half - far below scheduled demand levels - during peak arrival periods each day. The dissipation of clouds in the approach zone typically occurs during late morning. Since there is a very high demand for arrival capacity during this period, the precise timing of the transition to visual approaches has a significant impact on the return to full capacity operations. Better forecasts result in more efficient utilization of available airport arrival capacity.

The AAZCS has helped the Center Weather Service Unit (CWSU) provide better senvices to the FAA and the aviation community in the following ways:

- The AAZCS provides more accurate and complete real-time observations by providing live and continuous pictures. Before the AAZCS, the CWSU was dependant on hourly observations. With minute-tominute changes in weather affecting side by operations, this was insufficient.

- AAZCS provides a better understanding of the weather factors that are affecting visual observations. The observations are at the airport but the important 


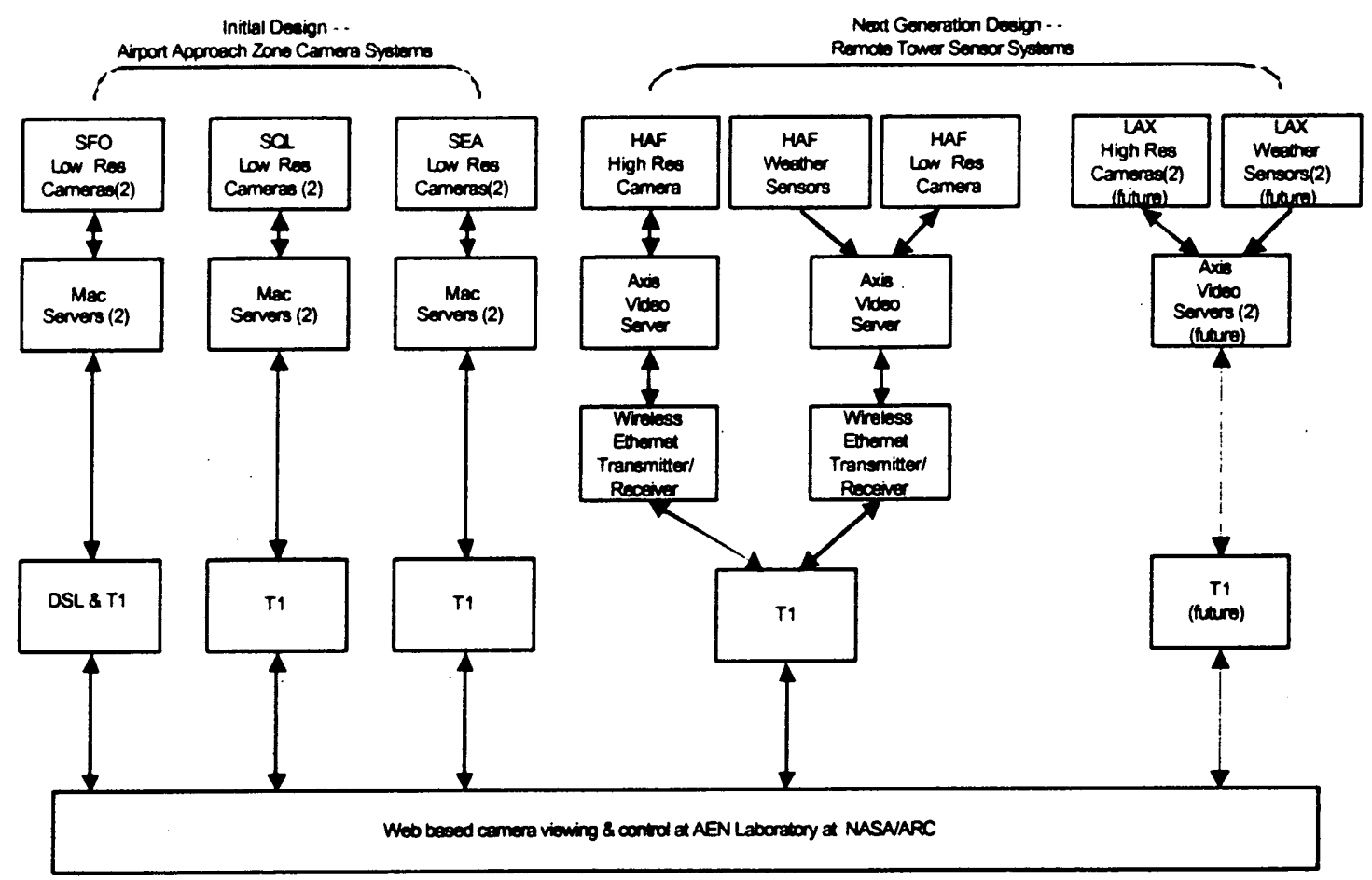

Figure 1: Overall configurations of the AAZCS and RTSS

area of concern is over the approach zone that extends from the airport to a distance 5-10 miles away. There are times when the airport is clear but the aircraft cannot see the airport because of clouds on the final approach zone. For example, at SFO foggy ocean air drifts into the SFO approach zone via the Crystal Springs Gap. The CWSU never realized this was a problem until they saw it on the cameras.

- The AAZCS supports collaborative efforts by providing the same timely information to decision makers at the FAA Air Traffic Control System Command Center and to the Airlines and other NOANNWS forecast offices that the CWSU at the Oakland ARTCC has access to. Thereby improving the probability of arriving at a credible, collaborative forecast that everyone can agree on.

The AAZCS consists of remote controlled cameras located on top of SFO and the SQL Traffic Control Towers. The cameras are controlled by the NOAA CWSU located at the Oakland Air Route Traffic Control Center (ARTCC) and are accessible via a secure web site. Figure 2 shows the locations of SFO, SQL and the CWSU at the Oakland FAA ARTCC. The AAZCS includes cameras that are able to provide a 220-degree field of view with rotate, zoom and tilt capabilities. Figure 3 shows one of two remote controlled camera systems used in AAZCS at SFO (a similar system is also installed at SQL). Figure 4 shows the Sony Pan/Tiltzoom color video camera each contained within the outdoor dome visible in Figure 3. The NOAA weather desk observer is able to see the sky condition as it is changing thereby providing a real-time evaluation of the conditions ang the approach zone of
SFO and SQL. The timing of forecasting weather changes in the final approach zone is very critical to airport operations and can result in savings of both time and revenue to the users.

Features of each outdoor dome (for the zoom/angle/pan camera) housing:

- The dome housing allows placement of each webcam with a panoramic view

- Housing is manufactured from UV plastic that will not yellow and is optically clear

- Tamper-resistant access

- The dome housing is available with a clear or tinted dome (one fstop light reduction)

- Temperature is controlled by heater and blower

- Wall mount bracket made from cast aluminum

- The housing top is formed from co-extruded 1/4" ABS plastic with UV stable exterior. The lower dome is formed from $1 / 8^{\prime \prime}$ cell cast acrylic

- Cables are threaded through a hole in the back of the mounting plate

- Weight: $3.25 \mathrm{lb}(1.47 \mathrm{~kg})$

Features of each low-resolution zoom/angle/pan camera:

- Sony Pan/TiltZZoom color video camera with remote control

- SiteZAP scriptable camera control application

- SiteZAP-Plugin WebSTAR server plug-in for total web access

- serial and composite cable for video cable

- Sony $12 x$ optical zoom with high-speed auto-focus 


\section{Two cameras}

mounted on top of the San Francisco International Airport tower

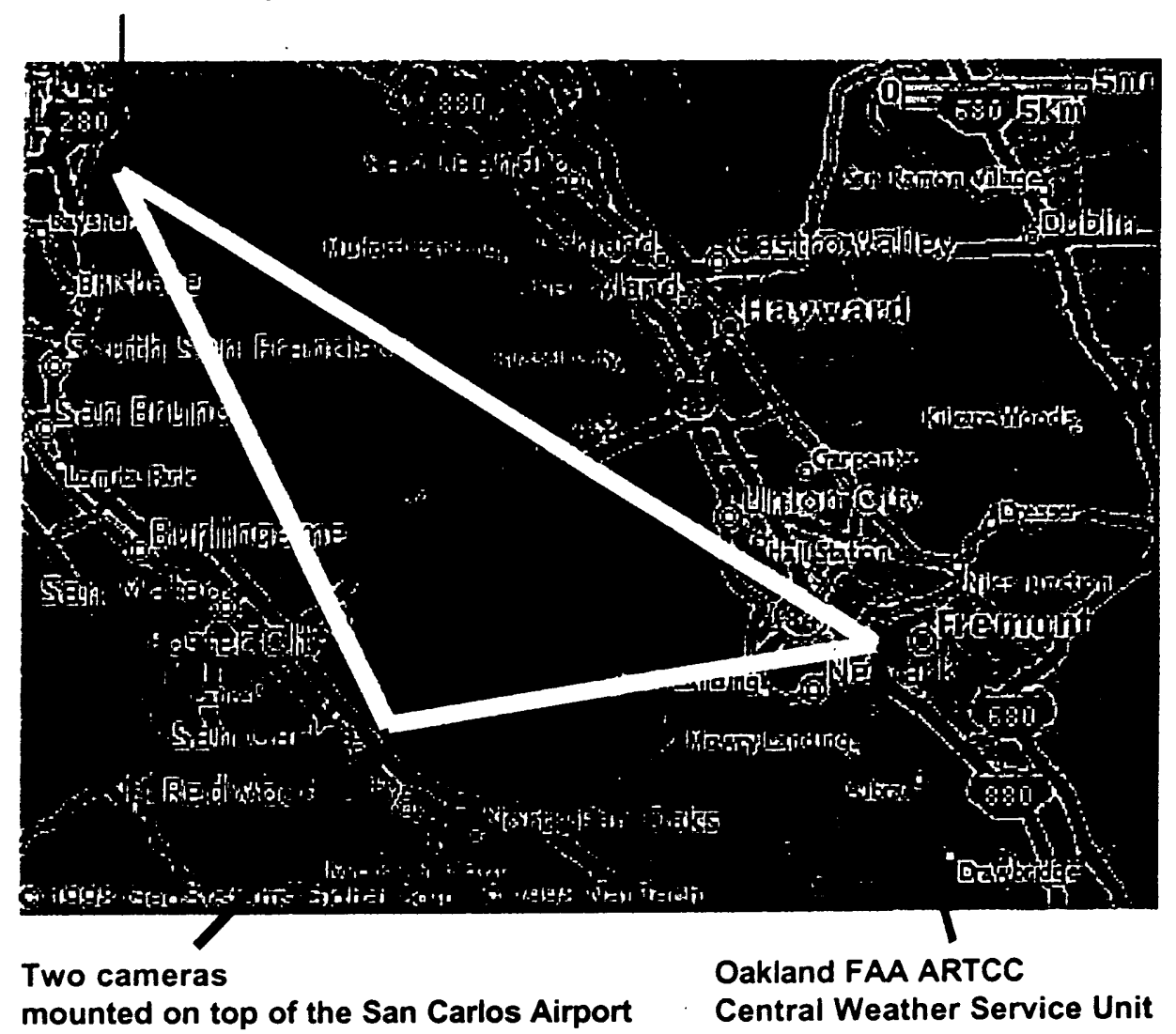

Figure 2: Locations of SFO, SQL \& Oakland FAA ARTCC

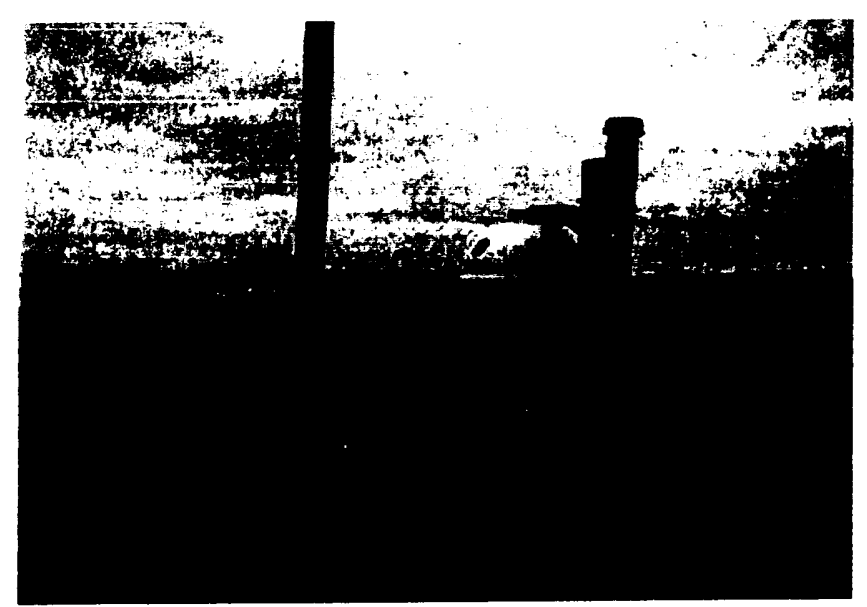

Figure 3: One of two remote controlled camera systems used in AAZCS at SFO (similar system is also installed at SQL)

- High-speed, wide range pan-tilt

- Remote control for easy access to all features

- Composite and S-Video outputs

- Connects to modem or printer port

- Powerful macro lens

- Pan/Tilt limit switches-keeps the camera in userspecified bounds

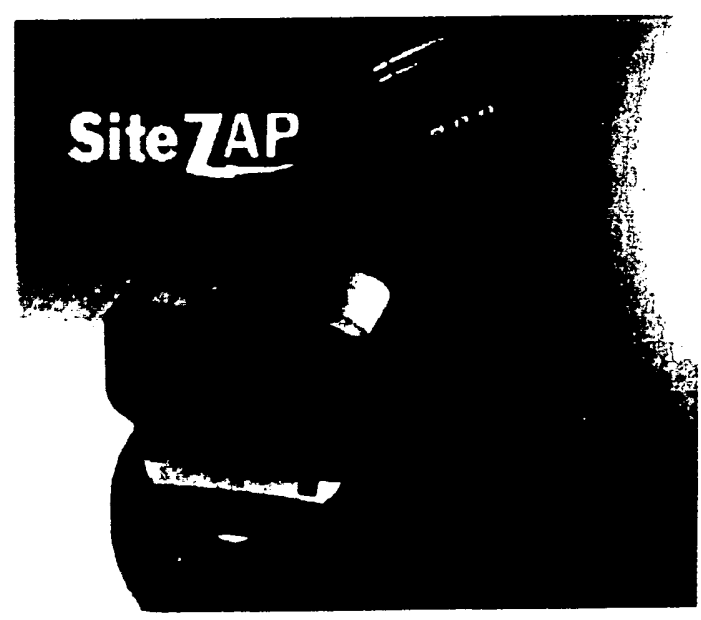

Figure 4: Sony PanTTiltZoom color video camera

- 6 position preset function (position, zoom, focus settings)

- Motion detector and auto-tracking features included

- Can be mounted in an optional outdoor dome enclosure

- Single-user SiteCam license for powerful image capture and video streaming 
Features of each AAZCS server:

- Macintosh CPU with Ethernet

- Color video input

- Internet connection with static IP address for streaming video

- A WebSTAR server

Upon the successful deployment of AAZCS at San Francisco Airport, the NASA Ames Research Center Aviation Surface Technologies Area team developed and deployed an AAZCS at the Seattle-Tacoma (SEA) International Airport in Washington State. The installation of this AAZCS involves a pair of remote controlled cameras located on top of SEA Traffic Control Tower (similar to the systems at SFO and SQL Airports).

\section{RTSS DEVELOPMENT}

A newly developed Remote Tower Sensor System (RTSS) represents a next generation design from the initial AAZCS design. In addition to secure web-based remote controlled camera systems, RTSS introduces real-time weather observations, remote sensing capabilities, portability, and deployment at remote and unmanned sites. RTSS can be utilized at both un-towered airports as well as at major airport hubs for synthetic vision augmentation for local as well as remote low/zero visibility operations. The goal of this research is to develop, deploy and demonstrate remotely operated cameras and sensors at several major airport hubs and un-towered airports for NASA, FAA, and NOAA research purposes. RTSS can provide real-time weather observations of airport approach zone in terms of forecasting the short-term onset and dissipation of low clouds.

RTSS COMPONENTS - RTSS will integrate and test airport sensor packages that will allow remote access to real-time airpoit conditions and aircraft status. Development of virtual tower systems that integrate realtime airport data to support operations for un-towered airports and for major hubs during low/zero visibility operations will become feasible. RTSS will provide more efficient airport arrival capacity operations and will result in savings of both time and revenue to its users. RTSS will include several key components that consist of sensor packages, camera systems, wireless communications, and real-time telemetry capabilities to provide conditions and aircraft status to support Smart Landing Facilities.

The initial installation of the RTSS will be at Half Moon Bay Airport. Figure 5 shows the overall RTSS configuration for HAF. Part of the system will be deployed on a tripod tower that can be easily repositioned. Figure 6 shows the HAF portable tripod structure. This prototype will consist of several key components as follows:

1. Ultrasonic wind sensor to provide wind speed and direction

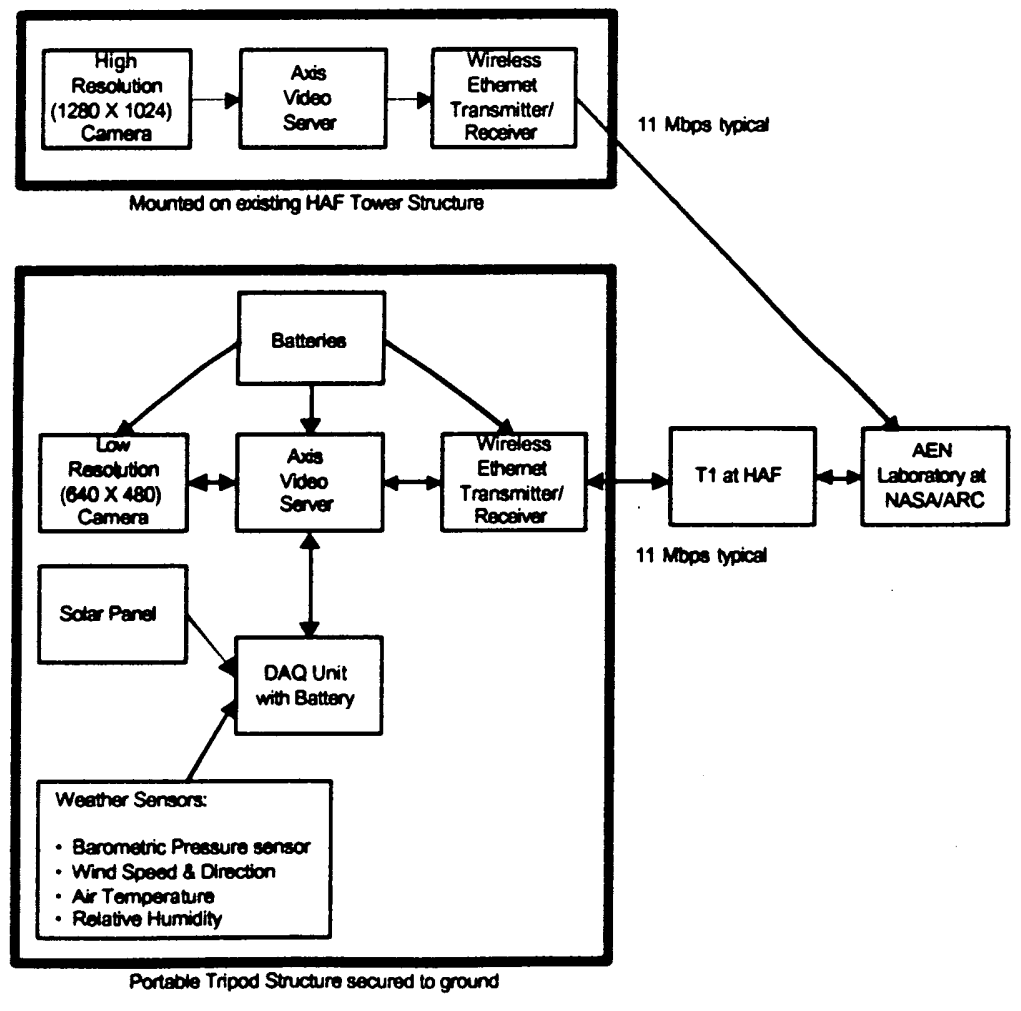

Figure 5: Overall RTSS Configuration at HAF 


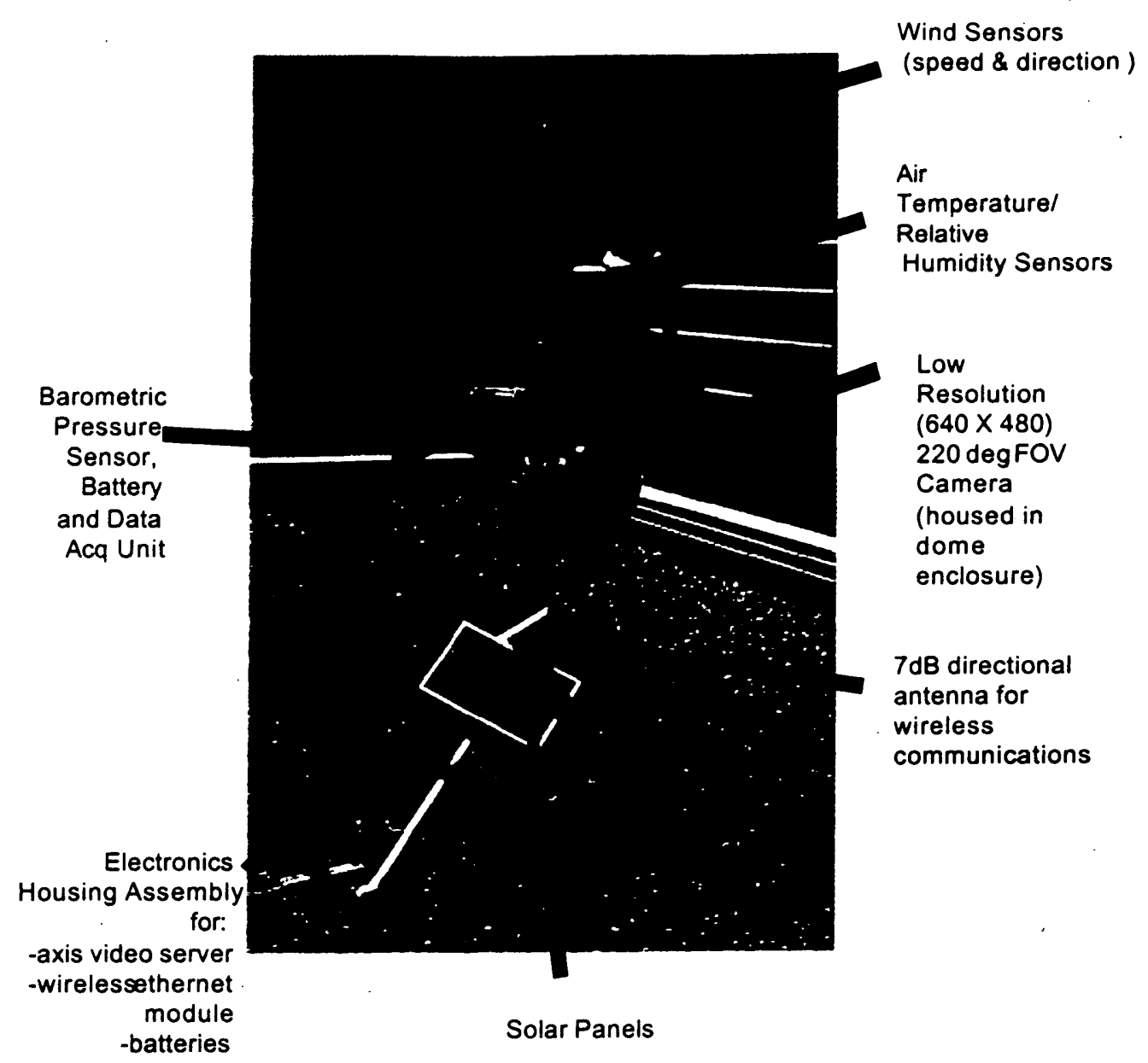

Figure 6: Development of HAF Portable Tripod Structure

2. Air temperature and relative humidity sensor

3. Barometric pressure sensor

4. Data acquisition (data logger unit) to collect sensor data

5. 9 foot $12.5 \mathrm{lb}$ portable tripod tower for easy deployment and repositioning

6. Embedded Web Video Server

7. Wireless Ethernet Module

8. Battery system to power the sensors and the devices

9. Solar panels

10. $14 \mathrm{~dB}$ Yagi directional antenna for wireless communications

11. Color PTZ camera

12. Dome enclosure to house PTZ camera

Also at HAF, as shown in Figure 7, will be a highresolution camera mounted on top an existing tower structure. The system at HAF will also be fielded at LAX in the future. Similar capability for the LAX terminal area and approach zone would be a great benefit for not only the Los Angeles (ZLA) CWSU, but also the ZLA Traffic Management Unit (TMU) and Southern Califomia Tracon. Here are a few scenarios where timely access to the cloud and weather images would benefit air traffic control (ATC) operations:

- STRATUS APPROACHING AT SHORELINE - Rapid movement of low clouds on the west edge of the field is not picked up on the official METAR/ASOS until about 10 minutes after the event. Time lapsed imagery would provide a significant lead time for remote ATC facilities.

- CLOUD COVER 3-7NM EAST OF LAX - Clouds in the approach zone frequently occur below $5000 \mathrm{msl}$ associated with the Palos Verdes convergence zone. Both the LAX official METAR and the downtown ASOS (CQT) may not see these clouds (vertically pointing laser ceilometer). Video cameras would verify the cloud cover and provide a timely update on the potential to regain visual approach when the clouds clear.

- THUNDERSTORM AND SHOWERS IN TERMINAL AREA - Rapidly changing ceilings, visibilities with convective cells passing through the airport terminal area and approach zone can be tracked easily with 1 minute or less updates using the time lapse from video cameras. METARJASOS reports only update with special observations for certain criteria and in any case take 10 minutes or longer before transmission to the CWSU and other ATC facilities.

Preliminary requirements and features for the LAX RTSS are as follows: 
- There is a requirement to have timely ( -1 minute) cloud morphology, visibility and wind data throughout the LAX terminal and approach arrival paths.

- There is a requirement to have real-time weather observation data throughout the LAX terminal and approach arrival paths in order to support forecasting the short-term onset and dissipation of low clouds.

- There is a requirement to integrate weather observation sources available in and around the LAX terminal area into a windowing software display. The primary sources include: video sky imagery, ASOS METAR obs, $1 \mathrm{~min}$ ASOS sensor data (winds, ceilometer, visibility sensor groups). Digital 1 minute RVRs, mesonet surface obs, and Low Level Wind Shear Alert System (LLWAS). Secondary sources include: NEXRAD radar, GOES satellite imagery, model grid analyses/forecasts, and vertical temperature/wind profilers.

- There is a requirement to have a software display system that facilitates the collaboration of decision makers who determine the weather-related arrival/departure configuration of LAX. Features that support this collaboration include: levels of access security, configurable alarm/alert thresholds of observational data, selectable time periods of animation of video imagery, interfaces to other data processing system and networks (AWIPS, Internet, ETMS), references to established official weather/operational aircraft criteria and remote system administration.

- There is a requirement to deliver data to the decision makers of LAX terminal operations in a way that efficiently uses communication bandwidth. Features that support this include: Local caching of observational data, user selection of data resolution (variable image resolution, selectable update rates of observations), client/server architecture.

VIRTUAL TOWER SYSTEMS - RTSSs will be part of dynamic virtual tower systems. In the case of an untowered airport, the information collected from a suite of RTSSs can be sent to a virtual tower system where a team of traffic controllers can utilize the information and perform real-time tower operations. Even for the case of major airport hubs, RTSS will play a significant role in providing real-time airport data to support operations

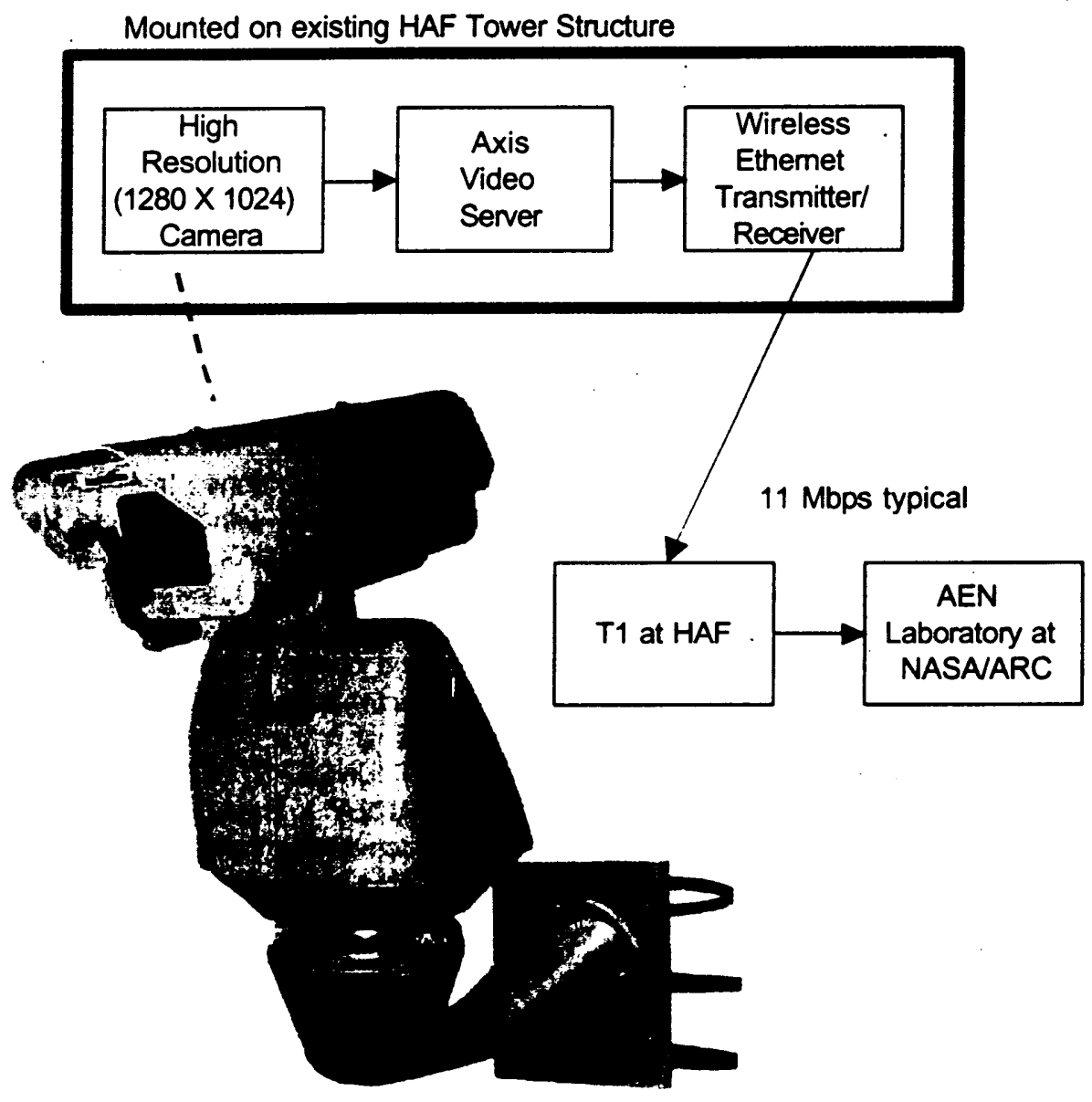

Pelco Camera 360 deg FOV

Figure 7: Development of HAF High Resolution System \& LAX (future) 


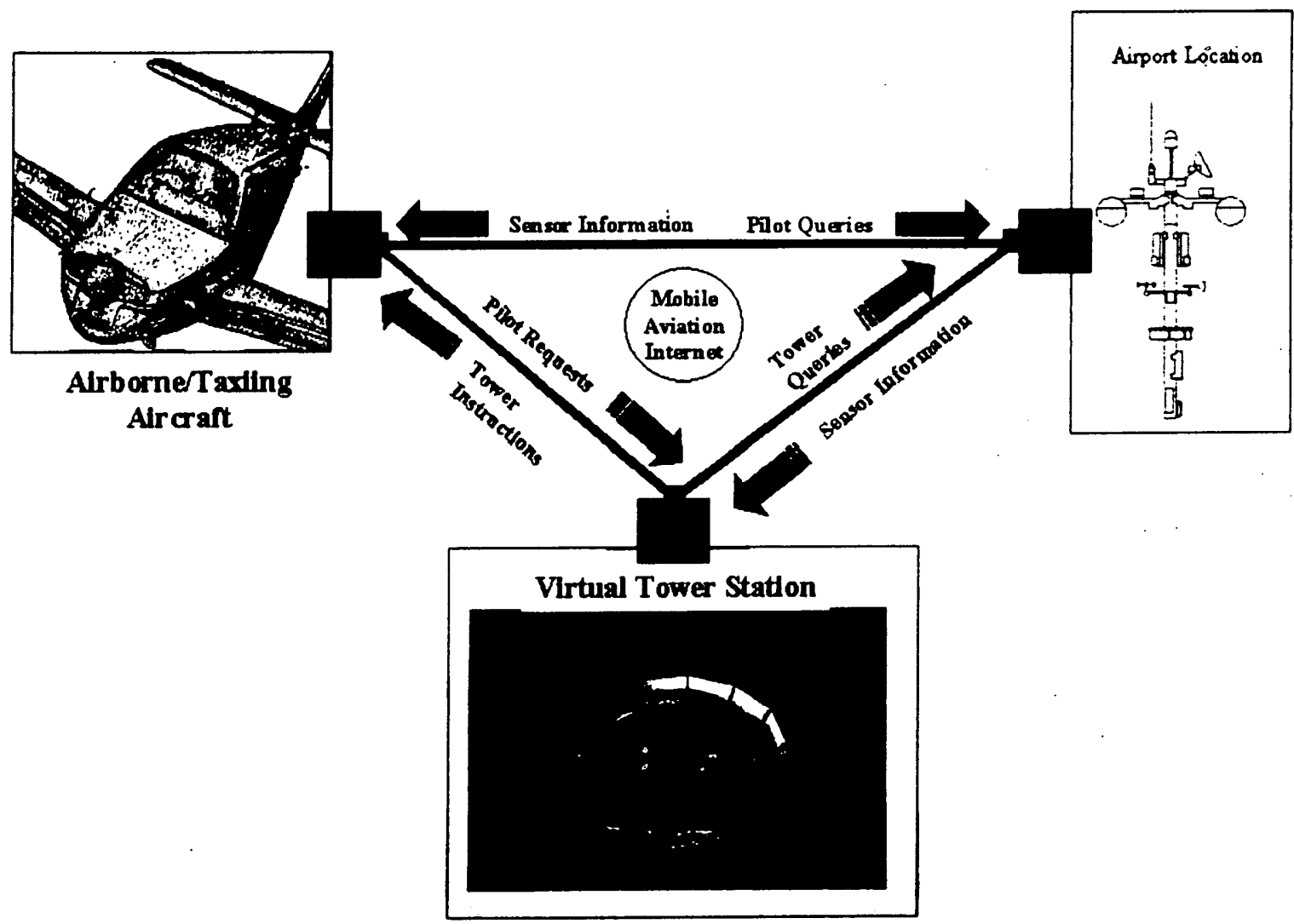

Figure 8: Dynamic Virtual Tower Support integrated with RTSS

during low/zero visibility scenarios.

The sensor and camera information will be integrated into virtual towers to provide real-time remote operations capability. Real-time data integration between pilots/aircraft and virtual tower stations will become feasible.

REAL-TIME AUTOMATED VISIBILITY MANAGEMENT SYSTEM - A real-time, automated visibility image management system has been developed for RTSS to track and monitor changing airportterminal conditions based on advanced image analysis and processing algorithms. The visibility management system predicts low to high visibility trends corresponding to suggestive zero to one incremental values for images captured by the camera systems. The images are captured and archived on a daily basis for every fifteen minutes time intervals. The visibility management system then processes captured images and updates the visibility versus time graphical plots (see Figure 9).

\section{CONCLUSION}

To improve weather-related delays and conditions at airports and unmanned towers, remote tower sensor systems have been developed and deployed by NASA ARC in collaboration with the FAA and. NOAA. This paper describes the initial proof-of-concept, AAZCS prototype design and the next generation Remote Tower Sensor System (RTSS) that will promote real-time weather observations and remote sensing capabilities at both remote and unmanned airport towers. Additional RTSS systems will be developed and deployed at both Half Moon Bay regional airport and Los Angeles International airport (LAX) in the near future. The RTSS systems have proved to be a vital research tool for NASA, the FAA, and NOAA and can result in saving both time and revenue to the end-users.

\section{ACKNOWLEDGMENTS}

The authors of this paper would like to acknowledge the collaborations with Walt Strach, Jr. Meteorologist-InCharge, NOAA at FAA, Oakland ARTCC; Bob Shiner, Air Traffic Operational Specialist, San Carlos and Half Moon Bay Airport; Mike Callahan. Traffic Management Coordinator, Seattle-Tacoma Intemational Airport; and Walt Rogers, Meteorologist-In-Charge, NOAA, Los Angeles (ZLA) CWSU.

\section{REFERENCES}

1. NASA Ames Research Center, Data Sharing Group, http://ic-www. arc.nasa.gov/datashare/RTSS.html

2. NASA Ames Research Center, RTSS Camera Page, http://ic-www. arc.nasa.gov/ic/projects/AAZCS 


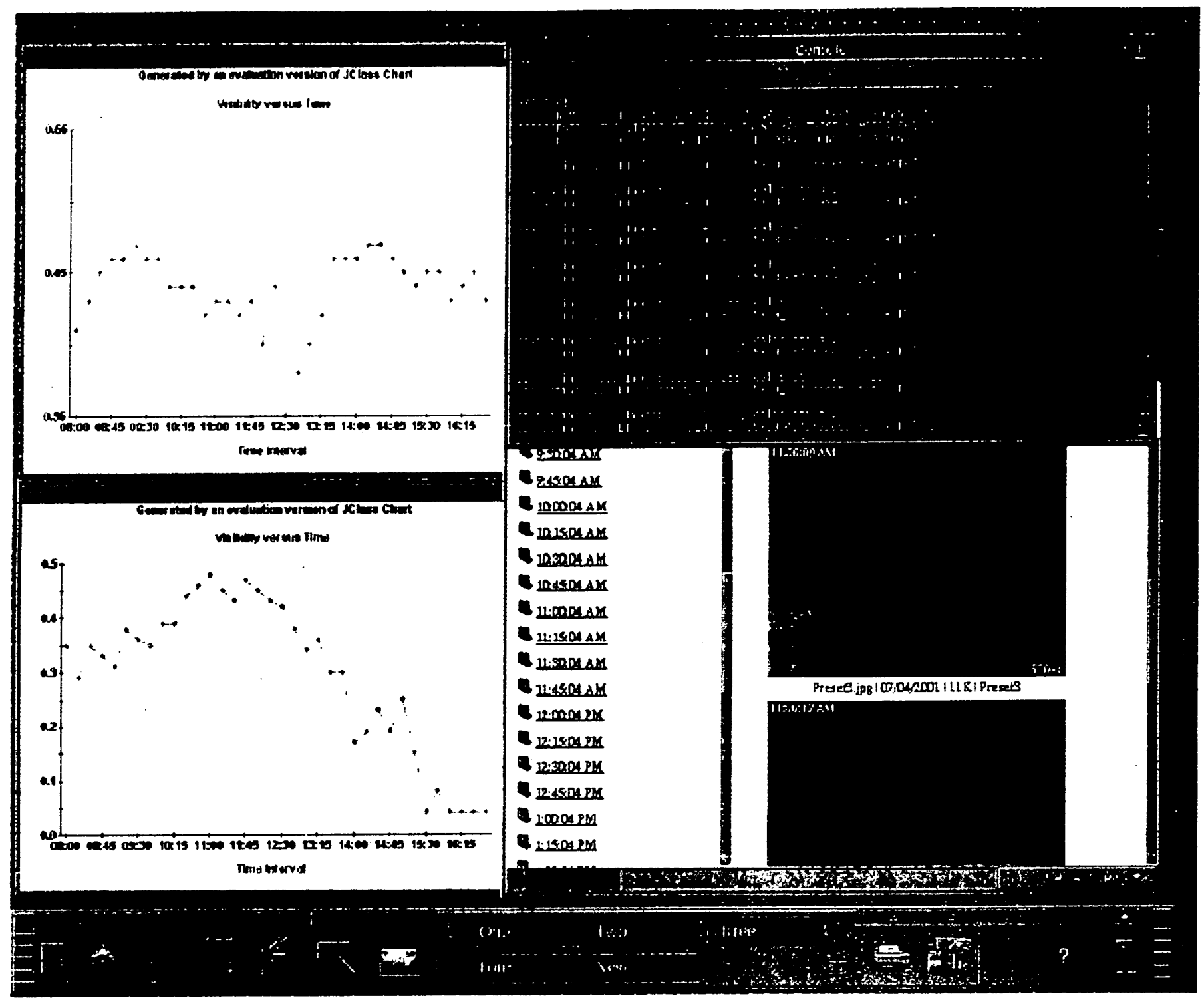

Figure 9: Real-time Automated Visibility Management System

\section{CONTACT}

Richard Papasin, NASA Ames Research Center, Mail Stop 269-2, Moffett Field, CA 94035-1000 rpaoasin@mail.arc.nasa.gov

Yuri Gawdiak, NASA Ames Research Center, Mail Stop 213-9, Moffett Field, CA 94035-1000 ygawdiak@mail.arc.nasa.gov

David A. Maluf, NASA Ames Research Center, Mail Stop 269-4, Moffett Field, CA 94035-1000 maluf@ptolemy.arc.nasa.gov

Christopher Leidich, NASA Ames Research Center, Mail Stop 269-4, Moffett Field, CA 94035-1000 cleidich@mail.arc.nasa.gov
Peter B. Tran, NASA Ames Research Center, Mail Stop 269-4, Moffett Field, CA 94035-1000 pbtran@mail.arc.nasa.gov

\section{DEFINITIONS, ACRONYMS, ABBREVIATIONS}

AAZCS: Airport Approach Zone Camera System ARC: Ames Research Center ARTCC: Air Route Traffic Control Center CWSU: Center Weather Service Unit FAA: Federal Aviation Administration LAX: Los Angeles International Airport NASA: National Aeronautical and Space Administration NOAA: National Oceanic Atmospheric Administration RTSS: Remote Tower Sensor System SEA: Seattle-Tacoma International Airport SFO: San Francisco international Airport SQL: San Carlos Airport 